\title{
Delayed flowering of guar plants (Cyamopsis tetragonoloba L. (Taub.)) in terms of metabolome
}

\author{
Teplyakova S.B. ${ }^{1 *}$, Shavarda A.L. ${ }^{2,3}$, Potokina E.K. ${ }^{1,2}$ \\ ${ }^{1}$ N.I. Vavilov All-Russian Institute of Plant Genetic Resources (VIR), St. Petersburg, Russia \\ ${ }^{2}$ St. Petersburg State University, St. Petersburg, Russia \\ ${ }^{3}$ Komarov Botanical Institute, RAS, St. Petersburg, Russia \\ *e-mail: serafima.teplyakova@mail.ru
}

Guar is an economically valuable crop used as a source to produce guar gum that is demanded by many industries. Guar is a short-day plant that performs best with shortened photoperiod (12-13 h). The date of flowering of guar is an important agro biological trait since it usually correlates with productivity of the crop. Identification of key molecules associated with the transition to generative phase of guar will help to detect genes controlling their biosynthesis and, accordingly, contributing to early flowering. Metabolomic profiling being a new approach of the system biology allows to reveal the internal factors determining the transition to the generative stage of guar. In our experiments 96 guar genotypes with different sensitivity to photoperiod from the VIR world collection were grown under long day conditions. For the photoperiodsensitive genotypes, the prolonged day light caused obstruction for switching the flowering program, which results in a strong delay of flowering. We detected differences in the metabolomic response between two guar groups: the first group of plants that started to flower without delay ("early flowering plants") and the second group of plants that delayed flowering significantly "delayed flowering plants". Metabolomic analysis showed that the metabolome of the "early flowering plants" and "delayed flowering plants" differs each from another by concentration of 7 molecules. We suggest to consider those as the key metabolites involved in the transition to flowering in guar.

Acknowledgements: This work was supported by grant No. 17-29-08027-ofi-m from the Russian Foundation for Basic Research and was carried out using the equipment of the Resource Center of the Scientific Park of St. Petersburg State University. 